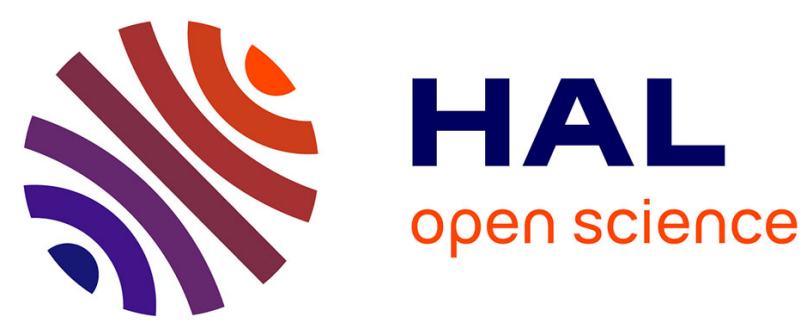

\title{
Why outcross? The abandon-ship hypothesis in a facultative outcrossing/ selfing fungal species
}

\author{
Océane Seudre, Alice Namias, Olivia Gardella, Guillaume Da Silva, \\ Pierre-Henri Gouyon, Manuela López-Villavicencio
}

\section{- To cite this version:}

Océane Seudre, Alice Namias, Olivia Gardella, Guillaume Da Silva, Pierre-Henri Gouyon, et al.. Why outcross? The abandon-ship hypothesis in a facultative outcrossing/ selfing fungal species. Fungal Genetics and Biology, 2018, 120, pp.1-8. 10.1016/j.fgb.2018.08.005 . hal-01992090

\section{HAL Id: hal-01992090 https: / hal.sorbonne-universite.fr/hal-01992090}

Submitted on 24 Jan 2019

HAL is a multi-disciplinary open access archive for the deposit and dissemination of scientific research documents, whether they are published or not. The documents may come from teaching and research institutions in France or abroad, or from public or private research centers.
L'archive ouverte pluridisciplinaire HAL, est destinée au dépôt et à la diffusion de documents scientifiques de niveau recherche, publiés ou non, émanant des établissements d'enseignement et de recherche français ou étrangers, des laboratoires publics ou privés. 


\title{
Why outcross? The abandon-ship hypothesis in a facultative outcrossing/ selfing fungal species
}

\author{
Océane Seudre $^{\mathrm{a}, 1}$, Alice Namias ${ }^{\mathrm{b}, 1}$, Olivia Gardella ${ }^{\mathrm{c}}$, Guillaume Da Silva ${ }^{\mathrm{a}}$, Pierre-Henri Gouyon ${ }^{\mathrm{a}}$, \\ Manuela López-Villavicencio ${ }^{\mathrm{a}, *}$
}

a Institut de Systématique, Evolution, Biodiversité (ISYEB), Muséum national d'Histoire naturelle, CNRS, Sorbonne Université, EPHE. CP 75005,57 rue Cuvier, 75005

Paris, France

b Département de Biologie, Ecole Normale Supérieure, PSL Research University, 75005 Paris, France

${ }^{\mathrm{c}}$ Facultad de Agronomía, Universidad de Buenos Aires, Capital Federal, Argentina

Keywords:

Haploid selfing

Homothallism

Sexual reproduction

Stress

Aspergillus nidulans

Microorganisms

\begin{abstract}
A B S T R A C T
Most species able to reproduce both sexually and asexually (facultative sexual species) invest more in sexual reproduction in stressful environment conditions. According to the abandon-ship hypothesis, plasticity for investment in sexual reproduction may have been selected in these species, allowing unfit genotypes to generate progeny carrying new advantageous allelic combinations. We tested this hypothesis in Aspergillus nidulans, a fungus able to reproduce asexually, or sexually, by outcrossing and/or haploid selfing (i.e. fusion of genetically identical haploid nuclei, causing immediate genome-wide homozygosity). We crossed various strains of $A$. nidulans in a non-stressful environment and a stressful environment containing a non-lethal dose of fungicide. Without stress, crosses preferentially generated haploselfed fruiting bodies, whereas stressful conditions significantly increased the outcrossing rate. Our results strongly support the abandon-ship hypothesis and suggest that, for parents with low fitness, the costs of investing in sexual reproduction may be compensated by the production of fitter progeny carrying beneficial allele combinations. Similarly, the progeny generated by outcrossing was less fit than that produced by haploid selfing in non-stressful environments, but fitter in stressful conditions, suggesting that outcrossing may have short-term advantages in stressful environments in A. nidulans.
\end{abstract}

\section{Introduction}

One key unresolved question in evolutionary biology concerns the maintenance of sexual reproduction despite its costs. In anisogamous sexual species, the females are at a disadvantage, as they suffer most of the cost of reproduction while providing only one copy of their genes to the progeny (Maynard Smith, 1978). Recombination between different genomes is also costly, as it may breaks allelic combinations and most of the new combinations generated are likely to be disadvantageous (Otto and Lenormand, 2002). Other costs, may also apply: sexual reproduction is usually slower than asexual reproduction; energy must be expended to find, recognize and/or attract a mating partner and there may be a risk of catching sexually transmitted parasites (Lehtonen et al., 2011). However, despite these costs, sexual reproduction is maintained in most eukaryotes suggesting that sex may provide benefits over different time scales that compensate for its costs (de Visser and Elena, 2007; Otto, 2009).
The long-term benefits of sexual reproduction include a lower susceptibility to extinction in sexual lines (Judson and Normark, 1996; Schwander and Crespi, 2009; Welch et al., 2000). Sexual reproduction confers advantages through recombination, decreasing the probability of fixation of harmful mutations, which tend to accumulate in asexual organisms (Muller, 1964). Sexual reproduction can also accelerate adaptation: the shuffling of genetic material may bring together favorable alleles carried by individuals from different genetic backgrounds (Muller, 1932).

Several possible short-term advantages have also been identified, mostly based on the production of a genetically diverse progeny. For example, according to the Red Queen hypothesis, in host-parasite interactions, both partners continually evolve in response to each other. Parasites are subject to strong selection for infection of the most common host genotypes in the population, and sexual reproduction of the host may facilitate rapid adaptation, by generating progeny with new genotypes that are more likely to escape infection (Howard and

\footnotetext{
* Corresponding author.

E-mail address: mlopez@mnhn.fr (M. López-Villavicencio).

${ }^{1}$ Both authors contributed equally to this work.
} 
Lively, 1994; Morran et al., 2011; Otto, 2009).

Other short-term advantages not related to the production of genetically diverse progeny have also been suggested. These advantages are based on mechanisms operating principally during meiosis. For example, sexual reproduction may ensure double-stranded DNA repair through the recombination of homologous chromosomes during meiosis (Bernstein and Johns, 1989; Bernstein et al., 2011). Sex may also help to eliminate RNA viruses, transposable or repeated sequences (including selfish DNA) in certain groups through the epigenetic silencing of these sequences during meiosis (Galagan and Selker, 2004; Rogers et al., 1986).

Many species, known as facultative sexual species, can reproduce both sexually and asexually. In these species, investment in these two modes of reproduction may vary over the lifetime of the organism. Such species are, thus, of particular interest for the study of sexual reproduction, because they can be used to decipher the environmental conditions selecting for an increase in the investment in sex (Hadany and Otto, 2007).

In general, the most facultative sexual species engage in sexual reproduction when confronted with various stresses (see (Agrawal et al., 2005; Hadany and Otto, 2007) for reviews). This suggests that sexual reproduction may be triggered by stressful environmental conditions in those species in which there has been selection for facultative sex.

Various hypotheses have been put forward to explain the increase in sexual reproduction in stressful conditions. According to the abandonship hypothesis, for example, sexual reproduction rates increase in stressful environments as a way of enabling individuals with ill-adapted genetic backgrounds to produce offspring carrying new allelic combinations, through recombination between two different genetic backgrounds. In stressful environments, ill-adapted individuals would be expected to switch to sexual reproduction, whereas adapted individuals are more likely to invest in growth and/or asexual reproduction (Agrawal et al., 2005; Hadany and Otto, 2007; Otto, 2009). The theoretical basis of the abandon-ship hypothesis has been studied in detail, and this hypothesis has been proposed as the cause of the increase in sexual over asexual reproduction in the plant Trifolium repens when individuals are exposed to stressful environments with high levels of herbivory (Griffiths and Bonser, 2013).

Increasing sexual reproduction rates in stressful conditions may also enable the organism to obtain the other benefits of sex not directly related to the production of diverse progeny. For example, in the facultative green alga Volvox carteri and the yeast Schizosaccharomyces pombe, sexual reproduction increases under oxidative stress. In both cases, it has been suggested that this increase is an adaptive response to DNA-damaging effects, allowing DNA repair to occur during meiosis (Bernstein and Johns, 1989; Nedelcu et al., 2004; Nedelcu and Michod, 2003). For other facultative species, it has been proposed that an increase in sexual reproduction in stressful conditions results from a coupling of sexual reproduction and the production of sexual spores or sexual eggs (Aanen and Hoekstra, 2007; Gouyon, 1999). For example, in some fungal species (as soil fungi or fungal food spoilers) produce heat-resistant sexual spores while asexual spores usually have little heat resistance (Dijksterhuis, 2007).

Here, we investigated the increase in sexual investment in facultative species in stressful environmental conditions. We were particularly interested in determining whether this increase enabled individuals facing stressful conditions to shuffle their genomes and generate fitter progeny (as proposed by the abandon-ship hypothesis) or to benefit from other advantages of sex associated with sex but not with the production of variable progeny.

We used the ascomycete fungus, Aspergillus nidulans as an experimental model. $A$. nidulans has an extended haploid life cycle, and sexual reproduction is achieved by the fusion of two vegetative haploid nuclei. This species is homothallic, so fusion does not require genetic differences between nuclei for mating compatibility. In homothallic species, syngamy is possible between genetically different vegetative nuclei produced by two different individuals (outcrossing), but also between two identical haploid nuclei originating from a common haploid progenitor, resulting in instant genome-wide homozygosity (Billiard et al., 2010; Billiard et al., 2012; Scazzocchio, 2006). This phenomenon is known as haploid selfing, or same-clone mating (Billiard et al., 2010; Billiard et al., 2012).

Previous studies in A. nidulans have shown that individuals increase their investment in sexual reproduction in stressful environments (Schoustra et al., 2010). Schoustra et al. (2010) used more than 50 genotypes of $A$. nidulans grown in three different stressful environments. They found that the different genotypes invested more resources in sexual reproduction in stressful conditions, particularly in environments in which their fitness was lower. In this study, genotypes were grown alone, so the increase in sexual reproduction was entirely due to haploid selfing, and the benefits of sex in this case were not linked to the production of a diverse progeny. Nevertheless the authors suggested that fusion tends to occur preferentially between genetically different nuclei in A. nidulans growing in natural conditions. Then, with available potential mating partners, increased investment in sexual reproduction would also increase genetic diversity in the offspring (Schoustra et al., 2010).

Since $A$. nidulans can reproduce by either outcrossing or haploid selfing, it is an ideal model for experimentation to address our questions and test whether the increased allocation to sex in stressful conditions enables to reshuffle genomes and create fitter progeny (as stated by the abandon-ship hypothesis) or if it is linked to advantages associated with sex, but not with the production of genetically diverse progeny. We tried to determine which of these hypotheses was most likely, by crossing different strains of $A$. nidulans in two environments: a regular culture medium and a stressful medium containing fungicide. We compared the proportion of sexual fruiting bodies (cleistothecia) produced by outcrossing with that produced by haploid selfing in the two environments. We also estimated the average number of ascospores produced depending on the type of cleistothecium (haploselfed or outcrossed) for each treatment.

According to the abandon-ship hypothesis, selection will favor individuals investing more in sexual reproduction in stressful conditions (as these individuals will benefit from the production of progeny carrying different allelic combinations). The proportion of outcrossed progeny relative to the progeny produced by haploid selfing should therefore increase when crosses occur in a stressful environment Conversely, if selection operates favoring individuals where sex is linked to other benefits (as DNA repair), the proportion of outcrossed progeny should not differ significantly between stressful and nonstressful conditions.

Under the abandon-ship hypothesis, genome shuffling in sexual reproduction should allow maladapted individuals to combine their genomes and produce new adaptive combinations, potentially increasing the mean fitness of their offspring. We therefore tested the fitness of the progeny produced by outcrossing or haploid selfing in the stressful and non-stressful environments. If sexual reproduction rates in ill-adapted parents increase to produce progeny with different allelic combinations, we would expect the outcrossed progeny (carrying new allelic combinations) to have a higher mean fitness than the progeny produced by haploid selfing (produced by sexual reproduction, but genetically identical to the parental strain), particularly in stressful conditions.

\section{Materials and methods}

\subsection{Strains, media and culture conditions}

Aspergillus nidulans is a common filamentous fungus that produces dark green asexual spores (conidia) and sexual fruiting bodies (cleistothecia) containing meiotic ascospores. We used six different strains of A. nidulans in this study (see Table 1 for the origin, date of isolation and 
Table 1

Strains of Aspergillus nidulans used in this study.

\begin{tabular}{|c|c|c|c|}
\hline Strain Number & $\begin{array}{l}\text { Geographic Origin } \\
\text { (and year of isolation) }\end{array}$ & Characteristics & Color \\
\hline WG655 & $\begin{array}{l}\text { Laboratory of } \\
\text { Genetics, Wageningen } \\
\text { University }\end{array}$ & Auxotroph & Green \\
\hline WG656 & $\begin{array}{l}\text { Laboratory of } \\
\text { Genetics, Wageningen } \\
\text { University }\end{array}$ & Auxotroph & Yellow \\
\hline LCP1992 & Egypt (1968) & Wild type & Green \\
\hline LCP2640 & Belgium (1982) & Wild type & Green \\
\hline LCP4275 & Morocco (1998) & Wild type & Green \\
\hline $\begin{array}{l}\text { LCP5709 (Pontecorvo strain, } \\
\text { FGSC A4 strain, isolated } \\
\text { by Yuill, 1939) }\end{array}$ & UK (1939) & Wild type & Green \\
\hline
\end{tabular}

description of all the strains used). Four of these strains were collected in natural environments and are held in the LCP collection of the $\mathrm{Mu}$ séum National d'Histoire Naturelle de Paris. The other two strains were obtained from the collection of the Laboratory of Genetics at Wageningen University. One of these two strains (WG655) produces green conidia, and has a mutation of the niaD gene, a structural gene encoding the apoenzyme of nitrate reductase. The other one (WG656) produces yellow conidia and carries a mutation of the $c n x$ gene, affecting the synthesis of a cofactor of nitrate reductase. For this strain, color and metabolic mutation are located in different chromosomes (Cove, 1976). These two laboratory strains are auxotrophic, bearing two different mutations affecting proteins from the same metabolic pathway for nitrate reduction. Both strains are derived from LCP 5709, a wild strain isolated by Yuill (1939) that is also known as the Pontecorvo strain, FGSC A4 or CBS 112.46. This strain was the first A. nidulans strain to have its whole genome sequenced (Table 1; Galagan et al., 2005).

The color and auxotrophy of these two strains are considered neutral in terms of fitness, when grown on urea-supplemented medium. They have been maintained asexually under laboratory conditions for over 350 generations and have accumulated mutations independently (López-Villavicencio et al., 2013). The other strains have been stored in liquid nitrogen in the LCP collection since their isolation. We did not see major differences in fruiting body production between the strains when grown alone.

We tested the growth of all the strains used, to ensure that they were susceptible to acriflavine, the fungicide used in the experiments. We also confirmed that the growth of all the strains in the fungicide treatment was equally reduced.

Unless otherwise stated, the strains were grown on urea-supplemented minimal medium (MM), to which we added $2 \%$ sucrose as a carbon source. This medium allows optimal growth in this species. It consisted of $6.0 \mathrm{~g} \mathrm{NaNO}_{3}, 1.5 \mathrm{~g} \mathrm{KH}_{2} \mathrm{PO}_{4}, 0.5 \mathrm{~g} \mathrm{MgSO}_{4} \cdot 7 \mathrm{H}_{2} \mathrm{O}, 0.5 \mathrm{~g} \mathrm{KCl}$, $1 \mathrm{mg}$ of each of the following trace elements: $\mathrm{FeSO}_{4}, \mathrm{ZnSO}_{4}, \mathrm{MnCl}_{2}$ and $\mathrm{CuSO}_{4}$, and $15 \mathrm{~g}$ agar in $1 \mathrm{~L}$ of demineralized water at $\mathrm{pH}$ 5.8. The plates were always incubated at $37^{\circ} \mathrm{C}$ to allow fungal growth.

Crosses took place in two different media. One of the media, an oatagar medium (OA) consisting of $40 \mathrm{~g}$ oat flakes, $15 \mathrm{~g}$ agar in $1 \mathrm{~L}$ of demineralized water, supplemented with $5 \mathrm{~mL} / \mathrm{L}$ urea, was not stressful. All strains can grow on urea, whether auxotrophic of wildtype. We also used a stressful OA medium of identical composition but supplemented with a non-lethal dose of $5 \mu \mathrm{g} / \mathrm{mL}$ of the fungicide acriflavin (Schoustra et al., 2010).

Fitness of the progeny was assessed on two different growth media for both outcrossed and haploselfed progenies: a non-stressful minimal medium (MM) and a stressful fungicide-supplemented minimal medium (FMM) containing a non-lethal concentration $(5 \mu \mathrm{g} / \mathrm{mL})$ of acriflavin (Schoustra et al., 2010).

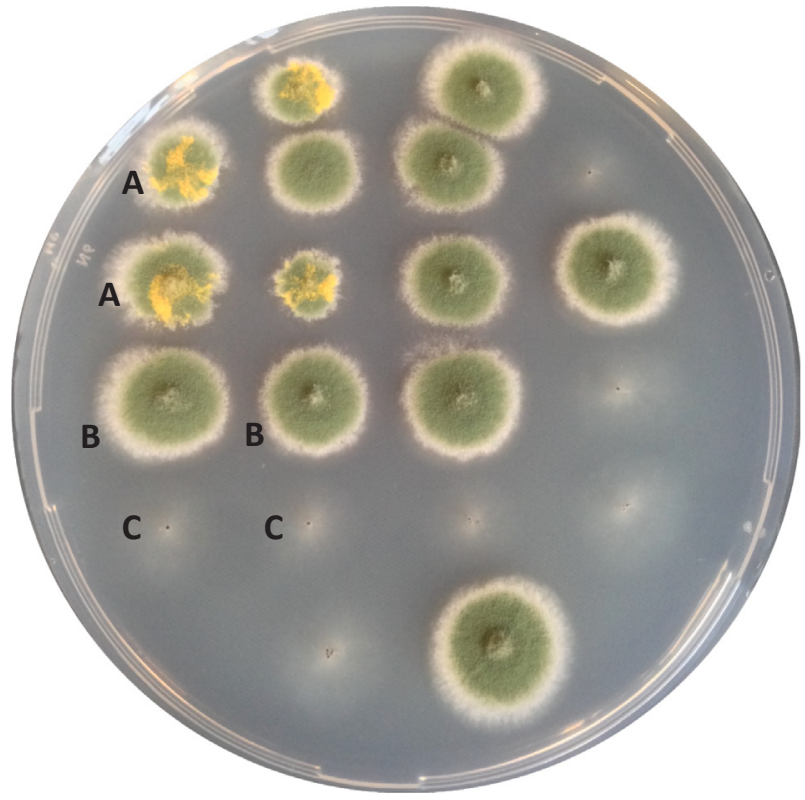

Fig. 1. Non-supplemented plates of minimal medium to determine which cleistothecia originated from outcrossing and which from haploid selfing. Cleistothecia were recovered from the crossing plates containing the WG656 (yellow, auxotroph) and $\times$ LCP5709 (green, oligotroph) strains. As no urea was added to the medium, only outcrossed cleistothecia and haploselfed cleistothecia from the wild strain LCP5709 were able to grow. Cleistothecia were crushed slightly before plating, to release ascospores. (A) Outcrossed cleistothecia produced by the cross between strains WG656 and LCP5709, with yellow and green germinating ascospores. (B) Single haploselfed cleistothecium produced by the wild strain LCP5709, with only green ascospores germinating. Ascospores produced by haploid selfing in this strain can germinate even in the absence of urea. (C) A haploselfed cleistothecium produced by the auxotrophic strain WG656, containing ascospores unable to germinate in urea-free medium. (For interpretation of the references to color in this figure legend, the reader is referred to the web version of this article.)

\subsection{Crosses}

We crossed the yellow strain (WG656) with the five different green strains (wild-type and auxotrophic). The use of a yellow mutant strain makes it easier to differentiate between outcrossed and haploselfed fruiting bodies (see below and Fig. 1). For each cross, we mixed equal quantities of spores $\left(10^{5}\right.$ spores) from each parental strain in a $1 \mathrm{~mL}$ Eppendorf tube of sterile water. We ensured that the suspension contained equal numbers of conidia from each of the two parental strains, by quantifying the conidia of each strain in a Malassez hemocytometer. Mixed suspensions were vortexed and for each cross, we spread $50 \mu \mathrm{L}$ of the fungal mixture onto normal OA (non-stressful) or OA supplemented with fungicide (stressful). For each cross, we prepared 10 plates of nonstressful $\mathrm{OA}$ and 10 of stressful OA. The crossing plates were then incubated for 15 days at $37^{\circ} \mathrm{C}$ in the dark.

\subsection{Estimation of phylogenetic distances}

In this species the genetic distance between parental strains may influence sexual compatibility and outcrossing rates. Hoffmann et al. (2001) showed that mating between genetically different strains produced mostly outcrossed progeny. On the other hand, Butcher showed that outcrossing between two closely related strains was not significantly higher than between two more genetically distantly strains (Butcher et al., 1972). We therefore estimated the phylogenetic distance between the different strains used for the crosses. We sequenced microsatellites previously developed for this species, with their nonrepetitive flanking regions (Christians and Watt, 2009; Hosid et al., 
Table 2

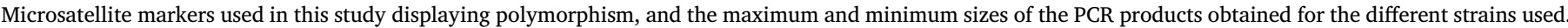

\begin{tabular}{|c|c|c|c|c|}
\hline \multirow[t]{2}{*}{ Marker name } & \multirow[t]{2}{*}{ Primer sequences $\left(5^{\prime} \rightarrow 3^{\prime}\right)$} & \multicolumn{2}{|c|}{ Size including flanking regions } & \multirow[t]{2}{*}{ Polymorphism } \\
\hline & & Min & Max & \\
\hline \multirow[t]{2}{*}{ NC8L1 } & TCA-GAG-GAT-CCA-GGA-CGA-CTA-G & 96 & 145 & $\mathrm{~N}$ \\
\hline & GAC-CTG-TGT-CAC-CTA-CGA-CTG-C & & & \\
\hline \multirow[t]{2}{*}{ NC6L1 } & CCA-TCA-CCA-TCC-GTA-CCT-CAC & 126 & 168 & $\mathrm{Y}$ \\
\hline & AAG-CTC-CAC-AGC-CGC-ATT-AC & & & \\
\hline \multirow[t]{2}{*}{ NC1L1 } & GCT-GGC-GAC-GAT-GAT-CCT-AC & 218 & 288 & $\mathrm{Y}$ \\
\hline & CAG-ATC-ATG-AAC-ACG-AGC-AAC-C & & & \\
\hline \multirow[t]{2}{*}{ An169 } & TGA-GCT-TAA-TGG-TGT-CGA-TG & 180 & 216 & $\mathrm{~N}$ \\
\hline & TCG-TGA-TTA-CCG-CAT-TTG-TA & & & \\
\hline \multirow[t]{2}{*}{ An164 } & AAA-TCA-CAA-GCC-GAT-AGC-TG & 69 & 138 & $\mathrm{Y}$ \\
\hline & ATC-ACC-CAA-GTT-CAA-GGA-AA & & & \\
\hline \multirow[t]{2}{*}{ An135 } & GTG-TAT-GCG-ATG-AGA-AGT-CG & 209 & 385 & $\mathrm{Y}$ \\
\hline & CAA-ATA-ATG-GGT-GGT-GAG-GA & & & \\
\hline \multirow[t]{2}{*}{ An89 } & CCG-TGT-CCC-GTT-TAA-GTA-TG & 243 & 337 & $\mathrm{Y}$ \\
\hline & TTG-ACA-GTC-TTG-GAG-CCT-TC & & & \\
\hline \multirow[t]{2}{*}{ An75 } & TCT-TTT-ACG-TTG-CGA-TTG-TG & 584 & 814 & $\mathrm{Y}$ \\
\hline & CTT-TCG-ATT-TGG-GGT-TTG-AT & & & \\
\hline \multirow[t]{2}{*}{ An44 } & СТT-TAT-СCT-СCС-ACC-АAC-СT & 78 & 321 & $\mathrm{Y}$ \\
\hline & TAG-GCT-TCT-GCT-GAT-TCT-GG & & & \\
\hline \multirow[t]{2}{*}{ An17A } & GGT-TGC-TAT-GCC-TAC-ATG-CT & 175 & 183 & $\mathrm{Y}$ \\
\hline & CAC-ACT-GCA-CCA-CCC-ATC & & & \\
\hline \multirow[t]{2}{*}{ An6A } & TAA-CCC-GGT-GGA-CAG-TAG-AA & 286 & 446 & $\mathrm{~N}$ \\
\hline & GGA-CAA-TCG-CTG-ACC-TCT-TA & & & \\
\hline
\end{tabular}

2005).

DNA was extracted from fresh mycelium of single-genotype strains grown for three days on malt agar at $37{ }^{\circ} \mathrm{C}$. The Qiagen DNeasy Plant Mini Kit (Qiagen, Ltd. Crawley, UK) was used for DNA extraction and purification. We used the following microsatellites, previously shown to be polymorphic: NC1L1, NC6L1, NC8L1 (Hosid et al., 2005) and An6A, An17A, An44, An75, An89, An135, An164, An169 (Christians and Watt, 2009). The sequenced flanking regions obtained were edited and assembled with CodonCode aligner v1.3.4. (CodonCode Software) (see Table 2 for details).

Nucleotide alignments were generated for individual sequences, with T-coffee v. 5.05 (Notredame et al., 2000), with the default settings. We calculated genetic distances between strains with MEGA6.06 (Tamura et al., 2013), using two models: Kimura-2-parameter (K2P) and p-distance.

\subsection{Identifying outcrossed and haploselfed progeny}

We wanted to test if individuals could increase the production of outcrossed progeny under stressful conditions. An increase in outcrossed progeny can be attained in this species by increasing the proportion of outcrossed sexual fruiting bodies and/or by increasing the average number of ascospores per outcrossed fruiting body.

In order to measure the proportion of outcrossed and haploselfed cleistothecia, we collected cleistothecia from each sexual cross after 15 days of incubation. The size of the cleistothecia is correlated with progeny fitness in this species (López-Villavicencio et al., 2013). We therefore collected only large, well-developed cleistothecia. The mode of reproduction (outcrossing between the two strains or haploid selfing of each parental strain) was identified by cleaning every cleistothecium individually, by rolling it into agar to detach conidia, and then plating them on selective non-supplemented minimal medium (Fig. 1). We isolated a total of 200 cleistothecia for each cross, and split them into groups of 20 cleistothecia per Petri dish. Cleistothecia were incubated at $37^{\circ} \mathrm{C}$ for four days, to allow germination to occur. In this non-supplemented medium, the haploselfed progeny of auxotrophic strains could not germinate. To differentiate between the haploselfed fruiting bodies produced by wild-type strains and those produced by outcross between yellow and green strains, we selected only germinating cleistothecia with both yellow and green conidia (Fig. 1). For each cross, we recorded the number of outcrossed and haploselfed cleistothecia on each type of medium (stressful and non-stressful OA). The outcrossing rate was determined using the following formula:

Outcrossing rate $=$ Number of outcrossed progeny/(number of outcrossed progeny + number of selfed progeny from both parental strains).

The average number of ascospores per fruiting body was estimated for the crosses that successfully produced outcrossed and haploselfed cleistothecia. For each cross, we counted the average number of ascospores produced by haploselfed and outcrossed cleistothecia produced under each treatment (i.e control and fungicide media). Cleaned cleistothecia were individually crushed to release ascospores in an Eppendorf tube containing $100 \mu \mathrm{L}$ of a solution of distilled water containing Tween- $800.0005 \%$. We plated $10 \mu \mathrm{L}$ of this ascospore suspension in a petri dish containing minimal medium supplemented with sucrose, urea and Triton X-100 $(400 \mu / \mathrm{L})$. The addition of Triton X-100 reduces colony growth, facilitating the observation of individual colonies. Plates were incubated at $37^{\circ} \mathrm{C}$ for 3 days in order to allow ascospore germination and identify if cleistothecia were haploselfed (all the colonies of one color either green or yellow) or outcrossed (producing colonies of both colors). For each cross, we selected 7 cleistothecia of each type (haploselfed produced by each parental strain and outcrossed) and for each treatment (control and fungicide). The number of ascospores was estimated via serial dilutions and plate counts on urea-Triton $\times$ minimal medium after 3 days of incubation at $37^{\circ} \mathrm{C}$.

\subsection{Isolation of outcrossed and haploselfed progeny and fitness assays}

Fitness was estimated as the diameter of the colony (MGR-mycelial growth rate), which is considered a good estimator of fitness for filamentous fungi (Pringle and Taylor, 2002).

We compared the fitness of outcrossed and haploselfed progenies from successful crosses in two different sets of environmental conditions: (1) a non-stressful minimal medium (MM) supplemented with $2 \%$ sucrose, and (2) a stressful fungicide minimal medium (FMM) supplemented with $2 \%$ sucrose and a non-lethal concentration $(5 \mu \mathrm{g} / \mathrm{mL})$ acriflavin (Schoustra et al., 2010).

Outcrossed progenies were recovered from the crossing plates. The 
germinating spores from cleistothecia identified as resulting from outcrossing (see above), were collected in $1 \mathrm{~mL}$ of sterile water in an Eppendorf tube. Single-spore isolations were systematically performed by serial dilution, to guarantee the presence of single isolated individuals. Haploselfed progenies were recovered from cleistothecia plates containing a single parental strain.

For each cross, we collected 15 outcrossed cleistothecia, when possible. We then isolated five individuals per cleistothecium. We recovered five haploselfed cleistothecia for each parental strain, and, similarly, isolated five individuals per cleistothecium.

For each isolated individual (outcrossed or haploselfed) we used $5 \mu \mathrm{L}$ of a suspension of conidia containing $10^{5}$ spores to inoculate the center of a Petri dish, along with an identical duplicate. Individuals were then incubated for six days at $37^{\circ} \mathrm{C}$.

For the fitness assays we needed to assure that only ascospores (sexual progeny) was measured and that sexual progeny were not mixed with conidia (asexual progeny) that would remain attached to the cleistothecium surface. Rolling the cleistothecia in agar is a wellaccepted cleaning technique and we were able to see the clean shiny cleistothecium wall under the microscope. In addition, we performed preliminary tests for checking that no conidia remained among isolated ascospores with this method.

Cleistothecia from the crosses between yellow and green strains were cleaned and individually crushed in $50 \mu \mathrm{L}$ of sterile water. The suspension was plated on urea-triton-supplemented MA medium and was incubated for 3 days at $37^{\circ} \mathrm{C}$ to allow germination of ascospores and of any conidia that would have remained attached to cleistothecia. When crushed cleistothecia resulted from haploid selfing of one of the parents used in the cross, all the small colonies observed should be of the same color (either yellow or green). In the case of outcrossed cleistothecia, we expected equal numbers of yellow and green colonies resulting from germinating ascospores. If some conidia produced by the green or yellow strains grown together on the crossing plates would have remained attached to the cleistothecium, we would expect in the case of haploselfed cleistothecia, a majority of colonies of a given color (resulting from ascospores contained in the cleistothecium), together with a small proportion of colonies of the alternative color (resulting from contaminant conidia from the strain that did not contribute this progeny). In the analyzed progeny, we observed only cases with either $100 \%$ of germinating spores of a single color, or balanced color ratios, but never highly skewed color ratios, indicating that no conidia remained in the germinating sexual ascospores.

\subsection{Statistical analyses}

Normality of the residuals and homoscedasticity of data were tested using Shapiro-Wilk's test.

To study differences in the fitness of the parental strain and the effect of the fungicide on the different fungal strains used for the crosses, we tested the differences in the size of the colony after 5 days of incubation in two different treatments (control or fungicide $5 \mu \mathrm{g} / \mathrm{mL}$ ) for the different strains using a Two-way ANOVA with the squared fitness (MGR) as the dependent variable, and strain and treatment as the independent variables.

Differences in the average number of ascospores between haploselfed (produced by each parental strain) and outcrossed cleistothecia in fungicide and non-fungicide medium were estimated using a twoway ANOVA with number of ascospores as the dependent variable; and type of cleistothecia (outcrossed or haploselfed for each strain) and treatment (fungicide or non fungicide) as independent variables.

We assessed the effects of strain and mode of sexual reproduction (outcrossing or haploid selfing) on both media (regular MM and FMM), by performing two-way ANOVA for each medium, with fitness as the dependent variable, strain and mode of reproduction as independent variables, and a strain $\times$ mode of reproduction interaction term. The significance of differences between means was assessed in Tukey tests, with the lsmeans (Lenth, 2016) and multcompView packages, in $\mathrm{R}$ software.

We tested the abandon-ship hypothesis (i.e. that individuals invest more in outcrossing when grown on a stressful medium) by logistic regression analysis, since the samples sizes were small. We analyzed the effects of genetic distance between the strains crossed, the crossing environment and their interaction on the proportion of the progeny generated by outcrossing, using a generalized linear model (GLM), with a logit link function and a binomial distribution.

All statistical tests were performed in JMP 9 (SAS Institute, Cary, NC, 2003) and R 3.4.2 (Development Core Team, 2014).

\section{Results}

\subsection{Fitness of the different strains used for the crosses}

In MM supplemented with urea and sucrose, we found no significant differences in MGR between the parental strains $\left(F_{5,24}=1.7979\right.$, $p=0.1514$ ). All the strains were sensitive to the fungicide acriflavine. The MGR of the strains was significantly reduced in the presence of the fungicide compared to the control $\left(F_{1,24}=13803.08, p<0.001\right)$. No differences were found in sensitivity between the different strains used; the fungicide reduced the fitness of all the strains in a proportional way (non-significant interaction $F_{1,24}=0.51, p<0.76$ ).

\subsection{Phylogenetic distances}

Three of the 11 markers (NC8L1, An169 and An6A) were monomorphic (Table 2). Only three of the polymorphic markers displayed positive amplifications in the WG655 strain. This strain is an auxotroph and, like WG656, was derived from LCP5709 (the Pontecorvo strain) by random mutation and the selection of color and metabolic markers (Cove, 1976). The estimation of phylogenetic distances on the flanking regions of the three polymorphic microsatellites positively amplified in this strain revealed a very similar genetic background in the WG655 and WG656 strains (data not shown). We therefore decided to remove the WG655 strain from the dataset, limiting our analyses to the other strains. The distance matrices obtained with the Kimura-2-parameter (K2P) and $p$-distance models were similar. We therefore present here only the results for the K2P model (Table 3). The distance matrix showed that, for the WG656 strain (used in all crosses), pairwise phylogenetic differences were lowest for the LCP5709 and WG655 strains, followed by the LCP4275 and LCP2640 strains. LCP1992 was the strain with the greatest phylogenetic distance to WG656 (Table 3, Fig. 2). Outcrossing rate was correlated with genetic similarity, and the crosses presenting the highest degree of genetic similarity (WG656 × LCP5709 and WG656 $\times$ WG655) also displayed the highest outcrossing rates, on both stressful and non-stressful media (see below, Fig. 2).

\subsection{Outcrossing versus haploid selfing in stressful conditions}

Regardless of the strains involved, cleistothecia were visibly more numerous for crosses performed on fungicide OA medium than for crosses performed on regular non-stressful OA medium. Outcrossing

Table 3

Estimates of evolutionary divergence between sequences under the Kimura-2 model, using sequenced microsatellite flanking regions as markers: NC6L1, NC1L1, An164, An135, An89, An75, An44 and An17A. Evolutionary analyses were conducted in MEGA6.06.

$\begin{array}{lllll}\text { WG 656 } & & & & \\ \text { LCP 5709 } & 0.009 & & & \\ \text { LCP 4275 } & 0.011 & 0.012 & 0.008 & \\ \text { LCP 2640 } & 0.012 & 0.012 & 0.031 & 0.030 \\ \text { LCP 1992 } & 0.025 & 0.031 & \end{array}$


100

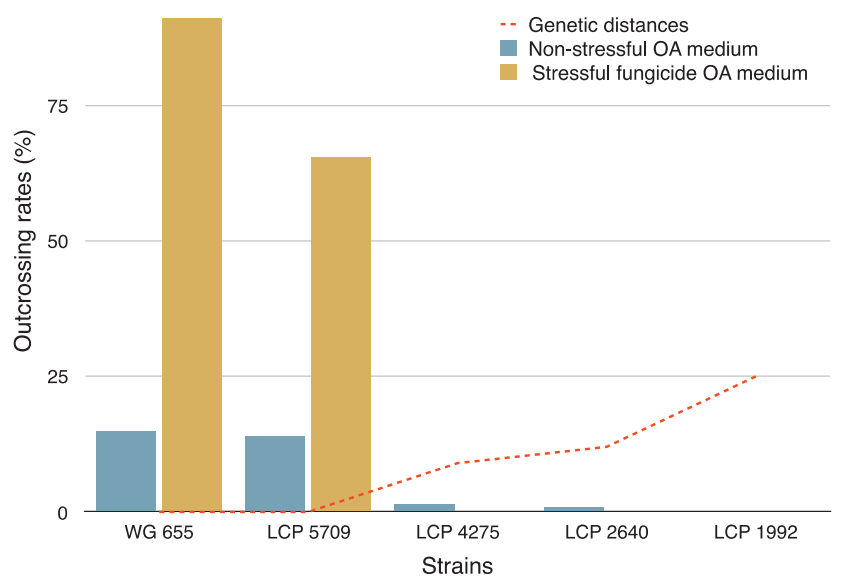

Fig. 2. Frequency of sexual fruiting bodies (cleistothecia) produced by outcrossing among the total number of fruiting bodies produced (haploselfed and outcrossed) in two different media (fungicide-containing stressful and nonstressful oat-agar medium), and genetic distances for the crosses between the WG656 strain and the other strains used in this study. Genetic distance was not measured for the WG655 strain (see the text), but, as WG655 and WG656 are both auxotrophic mutants derived from LCP5709, we considered them to be separated by a very small genetic distance, to illustrate our results.

rate was significantly influenced by both medium and genetic distance between the strains (GLM, $p<2.2 \mathrm{e}^{-16}$ for both factors). There was a significant interaction between medium and genetic distance (GLM, $p<2.2 \mathrm{e}^{-16}$ ). The outcrossing rate was significantly higher in stressful fungicide medium than on non-stressful medium (Fig. 2). Outcrossing rate decreased significantly with genetic distance, although this effect was medium-dependent, with a much stronger decrease on stressful medium.

The number of ascospores per cleistothecium was similar for haploselfed and outcrossed cleistothecia $\left(F_{3,63}=1.4566, p=0.2349\right)$. However, the crossing medium (stressful and non-stressful) had a significant effect in the number of ascospores in all cleistothecia types; outcrossed and haploselfed, contained more ascospores when crosses were produced in stressful medium $\left(F_{1,63}=6.3573, p=0.0142\right)$

\subsection{Fitness differences between outcrossed and haploselfed progeny}

We wanted to know whether the potential benefits of sexual reproduction were achieved through the production of genetically different progeny carrying potentially advantageous allelic combinations. We therefore compared the fitness of the offspring originating from outcrossing and those produced by haploid selfing. Despite the absence of differences in fitness between the parental strains, significant differences fitness were observed between outcrossed and haploselfed progenies, on both growth media (Fig. 3). Overall, individuals were significantly fitter on non-stressful minimal medium than on stressful fungicide-supplemented minimal medium.

On minimal medium, the fitness of the progeny was significantly influenced both by strain (ANOVA, $F_{5,281}=34.1174, p<2.2 \mathrm{e}^{-16}$ ) and mode of reproduction (outcrossing versus haploid selfing, ANOVA, $F_{1,281}=165.7054, p<2.2 \mathrm{e}^{-16}$ ). Haploselfed progenies had a significantly higher fitness and a lower fitness variance than outcrossed progeny, for all crosses (Tukey-tests, $p<1 \mathrm{e}^{-4,} p=0.0014$, $p=0.0023$ and $p<1 \mathrm{e}^{-4}$ for strains WG655, LCP2640, LCP4275 and LCP5709 respectively) (Fig. 3).

On stressful fungicide-containing minimal medium, both strain (ANOVA, $F_{5,275}=161.008, p<2.2 \mathrm{e}^{-16}$ ) and mode of reproduction (outcrossing versus haploid selfing; ANOVA, $d f=1, F_{1,275}=60.232$, $p=1.664 \mathrm{e}^{-13}$ ) had a significant effect on progeny fitness. There was also a significant interaction between strain and mode of reproduction (ANOVA, $F_{3,275}=19.517, p=1.681 \mathrm{e}^{-11}$ ).

The outcrossed progeny was significantly fitter than the haploselfed progeny only for the WG655 $\times$ WG656 cross (Tukey test, $p<1 \mathrm{e}^{-4}$ ). For all the crosses, fitness variance was higher for the outcrossed progeny than for the haploselfed progeny (Fig. 3)

\section{Discussion}

The primary aim of this study was to understand the selective pressures underlying the plasticity of investment in sexual reproduction in facultative sexual species. According to the abandon-ship hypothesis, selection favors individuals increasing sexual reproduction in environments where they are maladapted as shuffling two different genomes may generate offspring carrying new, fitter genetic combinations (Agrawal et al., 2005). Evidence supporting this hypothesis has been provided both theoretically (Agrawal et al., 2005; Hadany and Otto, 2007) and through experiments in the diploid plant Trifolium repens (Griffiths and Bonser, 2013). We used a homothallic fungal species that can reproduce by either outcrossing or haploid selfing to determine whether observed increases in sexual reproduction under stressful conditions were related to the generation of offspring with new and potentially fitter allelic combinations (as expected under the abandonship hypothesis) or whether this increase enabled individuals to benefit from advantages of sex related to meiosis.

Our results support the abandon-ship hypothesis for the crosses WG656 $\times$ WG655 and WG656 $\times$ LCP 5907 . We found that the percentage of the cleistothecia produced by outcrossing was significantly higher than the percentage of the cleistothecia resulting from haploid selfing when $A$. nidulans was grown under stressful conditions. The increase in outcrossing under stressful fungicide conditions was due to an increase in the production of outcrossed cleistothecia: the percentage of the sexual cleistothecia produced by outcrossing was about $15 \%$ in non-stressful medium, similar to previous results for A. nidulans (López-Villavicencio et al., 2013), whereas, in stressful fungicide-containing medium, outcrossing rates increased to $65 \%$ for one of the crosses and $90 \%$ for the other. The increased investment in sexual reproduction, associated with a higher proportion of outcrossed progeny strongly supports the hypothesis that sexual reproduction can provide a mean for ill-adapted individuals to increase the fitness of their offspring in a single episode of sexual reproduction, by reshuffling the genes present in the population.

The abandon-ship hypothesis also suggests that genome shuffling between individuals of low fitness may produce a fitter progeny carrying new adaptive alleles through sexual reproduction. The mean fitnesses of the outcrossed and haploselfed progenies are also consistent with this hypothesis. The fitness of the outcrossed progeny was significantly greater than that of the haploselfed offspring for one of the crosses in stressful conditions (fungicide-supplemented minimal medium), while the opposite situation was observed on regular minimal medium. In both media, fitness variance was significantly higher for the outcrossed than for the haploselfed progeny. This finding suggests that some short-term benefits may be achieved through the production of rare genotypes carrying new allelic combinations fitter than the parental genotypes (Colegrave et al., 2002; Greig et al., 1998). The generation of new genetic combinations in the progeny may, therefore, particularly important in stressful conditions (Colegrave et al., 2002).

We found that the proportion of cleistothecia produced by outcrossing was extremely low (less than $2 \%$ ) for three of the five crosses, in both stressful and non-stressful environments. In particular, crosses between the WG656 and LCP1992 strains produced no outcrossed progeny (Fig. 2). In this species, compatible systems based on non-self recognition alleles (het genes) have been described (Anwar et al., 1993; Dales and Croft, 1990), meaning that strains belonging to the same heterokaryon compatibility group carry the same set of het alleles and have usually similar or identical genetic backgrounds (Esser, 2016; 


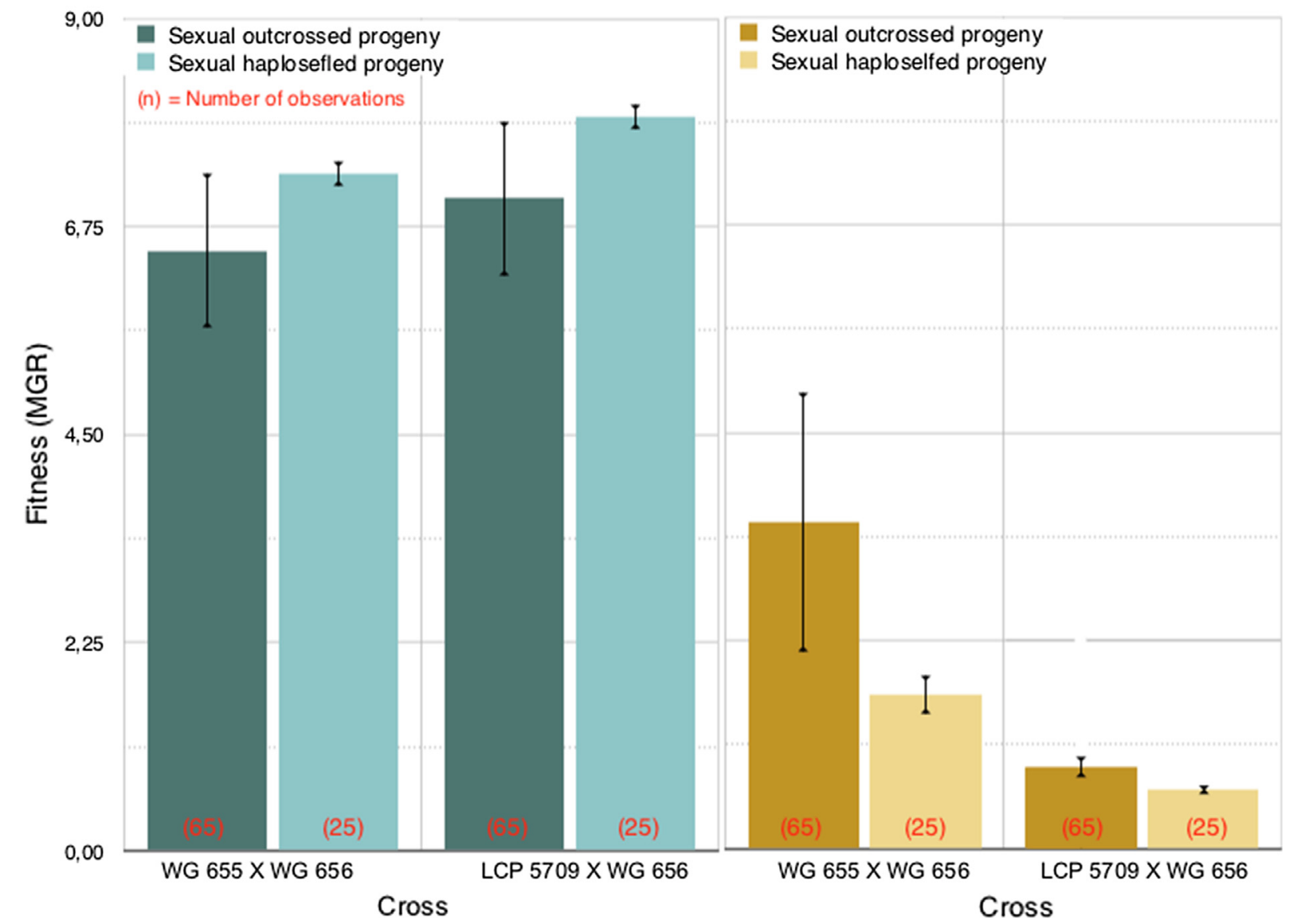

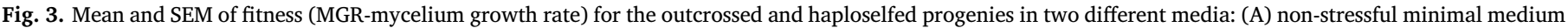
and (B) stressful fungicide-supplemented minimal medium. Progeny were produced for two different crosses: WG656 $\times$ WG655 and WG656 × LCP 5907 .

Geiser et al., 1994; Hoffmann et al., 2001). Butcher showed that heterokaryon incompatibility was not a barrier to outcross: outcrossing rate between two closely related strains was not significantly higher than between two more genetically distantly strains belonging to different het groups (Butcher et al., 1972). He also showed that strains could have different "outcrossing potential" and this varied significantly between heterokaryon incompatible, but not between heterokaryon compatible isolates (Butcher et al., 1972).

Our results for genetic distance showed a reduction in the outcross correlated with the genetic distance of the strains. The strain LCP1992 was the strain most different from WG656, in terms of genetic distance. By contrast, the LCP5709 and WG656 strains displayed the highest degree of phylogenetic similarity, and the highest outcrossing rate in both media (Fig. 2). Although the strains showing low or null outcrossing (i.e strains LCP1992 or LCP2640) could belong to a low outcrossing het group, in A. nidulans, the lower rates of outcrossing between more genetically distant strains may also be explained by the existence of prezygotic reproductive barriers. In ascomycete fungi, intersterility between closely related species is rare, both in sympatry and in allopatry, essentially because mating occurs between individuals sharing the same habitat after mycelial growth. Mating is therefore possible only between species adapted to the same environment (Le Gac et al., 2007). However, some examples of premating isolation between different species from the same habitat have been reported (Le Gac et al., 2007; Maclean and Greig, 2008). Interestingly, most studies on reproductive isolation in ascomycetes have focused on heterothallic species (Le Gac et al., 2007). In heterothallic ascomycetes, fusion between gametes is controlled by the mating type locus and fusion can only occur between haploids carrying different mating type alleles. In heterothallic, as opposed to homothallic, fungi, haploid selfing is prevented and sexual reproduction depends on the presence of a compatible partner (Billiard et al., 2010; Billiard et al., 2012). This difference may lead to differences in terms of mate choice and reproductive isolation between homothallic and heterothallic species. As sexual reproduction is not constrained by the presence of a compatible partner in homothallic species, individuals may be "choosier" in their selection of a partner, resulting in a greater propensity to undergo haploid selfing rather than outcrossing. In the homothallic species in which prezygotic reproductive isolation has been studied, species have been found to differ in their "choosiness", with an impact on the strength of prezygotic isolation. In two closely related homothallic species, Saccharomyces cerevisiae and $S$. paradoxus, prezygotic isolation mechanisms prevent hybridization between the two species. However, if no individuals of the same species are available, $S$. cerevisiae is choosier than $S$. paradoxus, suggesting that prezygotic isolation is stronger for $S$. cerevisiae (Maclean and Greig, 2008). The use of other homothallic species with well-known defined populations would undoubtedly generate interesting results concerning reproductive isolation and speciation in fungi.

\section{Conclusion}

Our study supports the abandon-ship hypothesis, which suggests that the plasticity of investment in sexual reproduction may have been selected in facultative species able to reproduce both sexually and asexually, because it generated progeny carrying new advantageous genetic combinations, particularly in stressful environments. Under non-stressful conditions, crosses preferentially resulted in haploselfed 
fruiting bodies, whereas stressful conditions triggered a switch to outcrossing for some of the pairs of strains considered, suggesting that, for poorly adapted parents, the cost of outcrossing may be compensated by the production of a fitter progeny carrying beneficial allelic combinations. Similarly, while the progeny produced by outcrossing was less fit than the progeny produced by haploid selfing in non-stressful environments, reverse was true in stressful conditions, suggesting that outcrossing can provide short-term advantages in stressful environments. Finally, we suggest that premating reproductive isolation mechanisms may operate in A. nidulans.

\section{Authors' contributions}

OS, AN, GDS and MLV preformed the crosses and fitness experiments. OG did the microsatellite sequencing and genetic distances analyses. PHG and MLV designed the experiments. OS, AN, OG and MLV analysed the data and wrote the paper. All authors read and approved the final manuscript.

\section{Acknowledgements}

We acknowledge Fons Debets and Marijke Slakhorst for providing the two auxotroph Aspergillus nidulans strains used in this study. We also thank Adrien Le Rouzic, Marie Saglio and Sandrine Lacoste for hard work and all the help with lab experiments. We are deeply grateful to Tatiana Giraud, Denis Roze and Claudio Scazzocchio for comments on previous versions of this manuscript. We also thank John Taylor for helpful discussion.

\section{Competing interests}

The authors have declared that no conflict of interests exists.

\section{References}

Aanen, D., Hoekstra, R., 2007. Why sex is good: on fungi and beyond. In: In: Heitman, J., Kronstad, J.W., Taylor, J.W., Casselton, L.A. (Eds.), Sex in Fungi: Molecular Determination and Evolutionary Implications ASM Press, Washington, DC, pp. 527-534.

Agrawal, A.F., Hadany, L., Otto, S.P., 2005. The evolution of plastic recombination. Genetics 171, 803-812.

Anwar, M.M., Croft, J.H., Dales, R.B.G., 1993. Analysis of heterokaryon incompatibility between heterokaryon-compatibility (H-C) groups $\mathrm{R}$ and $\mathrm{Gl}$ provides evidence that at least 8 Het loci control somatic incompatibility in Aspergillus nidulans. J. Gen. Microbiol. 139, 1599-1603.

Bernstein, C., Johns, V., 1989. Sexual reproduction as a response to H2O2 damage in Schizosaccharomyces pombe. J Bacteriol. 171, 1893-1897.

Bernstein, H., Bernstein, C., Michod, R.E., 2011. Meiosis as an evolutionary adaptation for DNA repair. In: In: Kruman, I. (Ed.), DNA Repair InTech Publ, Rijeka, Croatia, pp. 357-382.

Billiard, S., López-Villavicencio, M., Devier, B., Hood, M., Fairhead, C., Giraud, T., 2010. Having sex, yes, but with whom? Inferences from fungi on the evolution of anisogamy and mating types. Biol. Rev. 86, 1-22.

Billiard, S., López-Villavicencio, M., Hood, M., Giraud, T., 2012. Sex, outcrossing and mating types: unsolved questions in fungi and beyond. J. Evol. Biol. 25, 1020-1038.

Butcher, A.C., Croft, J., Grindle, M., 1972. Use of genotype-environmental interaction analysis in the study of natural populations of Aspergillus nidulans. Heredity 29, 135-149.

Christians, J.K., Watt, C.A., 2009. Mononucleotide repeats represent an important source of polymorphic microsatellite markers in Aspergillus nidulans. Mol. Ecol. Reso. 9, 572-578.

Colegrave, N., Kaltz, O., Bell, G., 2002. The ecology and genetics of fitness in Chlamydomonas. VIII. The dynamics of adaptation to novel environments after a single episode of sex. Evolution 56, 14-21.

Cove, D.J., 1976. Cholorate toxicity in Aspergillus nidulans: the selection and characterisation of chlorate resistant mutants. Heredity 36, 191-203.

Dales, R.B., Croft, J.H., 1990. Investigation of the het genes that control heterokaryon incompatibility between members of heterokaryon-compatibility (h-c) groups A and G1 of Aspergillus nidulans. J. Gen. Microbiol. 136, 1717-1724.

de Visser, J.A., Elena, S.F., 2007. The evolution of sex: empirical insights into the roles of epistasis and drift. Nature Rev. Genet. 8, 139-149.

Dijksterhuis, J., 2007. Heat-resistant ascospores. In: In: Dijksterhuis, J., Samson, A.R. (Eds.), Food Mycology: A Multifaceted Approach to Fungi and Food CRC Press Taylor
\& Francis Group, Boca Raton, USA, pp. 101-117.

Development Core Team, R., 2014. A Language and Environment for Statistical Computing. R Foundation for Statistical Computing, Vienna, Austria ISBN 3-90005107-0.

Esser, K., 2016. Heterogenic incompatibility in fungi. In: In: Wendland, J. (Ed.), Growth, Differentiation and Sexuality. The Mycota, vol I. Springer International Publishing, pp. 103-131.

Galagan, J., Selker, E., 2004. RIP: the evolutionary cost of genome defense. Trends Genet. $20,417-423$.

Galagan, J.E., Calvo, S.E., Cuomo, C., Ma, L.J., Wortman, J.R., Batzoglou, S., Lee, S.I., Basturkmen, M., Spevak, C.C., Clutterbuck, J., Kapitonov, V., Jurka, J., Scazzocchio, C., Farman, M., Butler, J., Purcell, S., Harris, S., Braus, G.H., Draht, O., Busch, S., D'Enfert, C., Bouchier, C., Goldman, G.H., Bell-Pedersen, D., Griffiths-Jones, S., Doonan, J.H., Yu, J., Vienken, K., Pain, A., Freitag, M., Selker, E.U., Archer, D.B., Penalva, M.A., Oakley, B.R., Momany, M., Tanaka, T., Kumagai, T., Asai, K., Machida, M., Nierman, W.C., Denning, D.W., Caddick, M., Hynes, M., Paoletti, M., Fischer, R., Miller, B., Dyer, P., Sachs, M.S., Osmani, S.A., Birren, B.W., 2005. Sequencing of Aspergillus nidulans and comparative analysis with A. fumigatus and A. oryzae. Nature 438, 1105-1115.

Geiser, D.M., Arnold, M.L., Timberlake, W.E., 1994. Sexual origins of British Aspergillus nidulans isolates. Proc. Natl. Acad. Sci. 91, 2349-2352.

Gouyon, P.-H., 1999. Sex: a pluralist approach includes species selection (one step beyond and it's good). J. Evol. Biol. 12, 1029-1030.

Greig, D., Borts, R.H., Louis, E.J., 1998. The effect of sex on adaptation to high temperatures in heterozygous and homozygous yeast. Proc. R. Soc. B 265, 1017-1023.

Griffiths, J.G., Bonser, S.P., 2013. Is sex advantageous in adverse environments? A test of the abandon-ship hypothesis. Am. Nat. 182, 718-725.

Hadany, L., Otto, S., 2007. The evolution of condition-dependent sex in the face of high costs. Genetics 176, 1713.

Hoffmann, B., Eckert, S.E., Krappmann, S., Braus, G.H., 2001. Sexual diploids of Aspergillus nidulans do not form by random fusion of nuclei in the heterokaryon. Genetics 157, 141-147.

Hosid, E., Grishkan, I., Frenkel, Z., Wasser, S.P., Nevo, E., Korol, A., 2005. Microsatellite markers for assessing DNA polymorphism of Emericella nidulans in nature. Mol. Ecol. Notes 5, 647-649.

Howard, R.S., Lively, C.M., 1994. Parasitism, mutation accumulation and the maintenance of sex. Nature 367, 554-557.

Judson, O.P., Normark, B.B., 1996. Ancient asexual scandals. Trends Ecol. Evol. 11, 41-46.

Le Gac, M., Hood, M.E., Giraud, T., 2007. Evolution of reproductive isolation within a parasitic fungal species complex. Evolution 61, 1781-1787.

Lehtonen, J., Jennions, M.D., Kokko, H., 2011. The many costs of sex. Trends Ecol. Evol. $27,172-178$.

Lenth, R.V., 2016. Least-squares means: the R package lsmeans. J. Stat. Soft. 69, 1-33.

López-Villavicencio, M., Debets, A., Slakhorst, M., Giraud, T., Schoustra, S., 2013. Deleterious effects of recombination and possible nonrecombinatorial advantages of sex in a fungal model. J. Evol. Biol. 26, 1968-1978.

Maclean, C.J., Greig, D., 2008. Prezygotic reproductive isolation between Saccharomyces cerevisiae and Saccharomyces paradoxus. BMC Evol. Biol. 8, 1.

Maynard Smith, J., 1978. The Evolution of Sex. Cambridge University Press, Cambridge.

Morran, L., Schmidt, O., Gelarden, I., Parrish, R., Lively, C., 2011. Running with the Red Queen: host-parasite coevolution selects for biparental sex. Science 333, 216-218.

Muller, H.J., 1932. Some genetic aspects of sex. Am. Nat. 66, 118-138.

Muller, H.J., 1964. The relationship of recombination to mutational advance. Mut. Rese. 1, 2-9.

Nedelcu, A.M., Marcu, O., Michod, R.E., 2004. Sex as a response to oxidative stress: A twofold increase in cellular reactive oxygen species activates sex genes. Proc. R. Soc. B 271, 1591-1596.

Nedelcu, A.M., Michod, R.E., 2003. Sex as a response to oxidative stress: the effect of antioxidants on sexual induction in a facultatively sexual lineage. Proc. R. Soc. B 270 (Suppl 2), S136-S139.

Notredame, C., Higgins, D.G., Heringa, J., 2000. T-coffee: a novel method for fast and accurate multiple sequence alignment. J. Mol. Biol. 302, 205-217.

Otto, S.P., 2009. The evolutionary enigma of sex. Am. Nat. 174, S1-S14.

Otto, S.P., Lenormand, T., 2002. Resolving the paradox of sex and recombination. Nature Rev. Genet. 3, 252-261.

Pringle, A., Taylor, J.W., 2002. The fitness of filamentous fungi. Trends Microbiol. 10, 474-481.

Rogers, H.J., Buck, K.W., Brasier, C.M., 1986. Transmission of double-stranded RNA and a disease factor in Ophiostoma ulmi. Plant Path. 35, 277-287.

Scazzocchio, C., 2006. Aspergillus genomes: secret sex and the secrets of sex. Trends Genet. 22, 521-525.

Schoustra, S., Rundle, H.D., Dali, R., Kassen, R., 2010. Fitness-associated sexual reproduction in a filamentous fungus. Curr. Biol. 20, 1350-1355.

Schwander, T., Crespi, B., 2009. Twigs on the tree of life? Neutral and selective models for integrating macroevolutionary patterns with microevolutionary processes in the analysis of asexuality. Mol. Ecol. 18, 28-42.

Tamura, K., Stecher, G., Peterson, D., Filipski, A., Kumar, S., 2013. MEGA6: molecular evolutionary genetics analysis version 6.0. Mol. Biol. Evol. 30, 2725-2729.

Welch, M., David, B., Meselson, M., 2000. Evidence for the evolution of bdelloid rotifers without sexual reproduction or genetic exchange. Science 1211-1215.

Yuill, E., 1939. Two new Aspergillus mutants. J. Bot. 77, 174-175. 